Supporting Information for

Strategy to tether organometallic ruthenium-arene anti-cancer compounds to recombinant human serum albumin

Wee Han Ang, ${ }^{\mathrm{a}}$ Elisa Daldini, ${ }^{\mathrm{a}}$ Lucienne Juillerat-Jeanneret, ${ }^{\mathrm{b}}$ and Paul J. Dyson ${ }^{\mathrm{a} *}$

a. Institut des Sciences et Ingénierie Chimiques, Ecole Polytechnique Fédérale de Lausanne (EPFL), CH-

1015 Lausanne, Switzerland

b. University Institute of Pathology, Centre Hospitalier Universitaire Vaudois (CHUV), CH-1011

Lausanne, Switzerland.

\title{
Experimental
}

All reagents were purchased from Acros Chemicals unless otherwise indicated. $\mathrm{RuCl}_{3} \cdot \mathrm{xH}_{2} \mathrm{O}$ was obtained from Precious Metals Online. Recombinant HSA was purchased as a 5\% solution in PBS (containing $4 \mathrm{mM}$ sodium caprylate and $4 \mathrm{mM}$ acetyltryptophan) from New Century Pharmaceuticals (Huntsville, Alabama, US). Succinyl HCl terephthalic hydrazine (SHTH) and benzoyl hydrazine were prepared according to literature procedures. ${ }^{1,2}$ The reactions were performed with solvents dried using appropriate reagents and distilled prior to use. NMR spectra were measured on a Bruker DMX 400, using $\mathrm{SiMe}_{4}$ for ${ }^{1} \mathrm{H},{ }^{13} \mathrm{C}$ and $\mathrm{H}_{3} \mathrm{PO}_{4}$ for ${ }^{31} \mathrm{P}$ as external standards at $20^{\circ} \mathrm{C}$. Positive mode nano-electrospray ionization mass spectra (nESI-MS) for synthesized compounds were recorded on a ThermoFinnigan LCQ Deca XP Plus quadrupole ion trap instrument on samples dissolved in water with ionisation energy set at $1.7 \mathrm{~V}$ and capillary temperature at $150^{\circ} \mathrm{C}$ as previously described. ${ }^{3}$ Elemental analyses were carried out at the Institute of Chemical Sciences and Engineering (EPFL). 
Synthesis of 1-methylamine-1,4-cyclohexadiene

The synthetic method was adapted from a reported procedure. ${ }^{4}$ Benzylamine $(20 \mathrm{ml}$, $179 \mathrm{mmol})$ and ethanol $(80 \mathrm{ml})$ were added to stirring liquid ammonia $(400 \mathrm{ml})$ at $-56^{\circ} \mathrm{C}$ under nitrogen. Metallic sodium (16 g, $696 \mathrm{mmol}$ ) was added in small portions and the reaction was stirred for a further $4 \mathrm{~h}$. On completion of the reaction, indicated by the persistent blue colour, the reaction mixture was quenched with excess solid ammonium chloride (80 g, $1.50 \mathrm{~mol})$. The ammonia was allowed to evaporate overnight, and the residue was dissolved in water $(300 \mathrm{ml})$ and extracted with diethyl ether (3 x $250 \mathrm{ml})$. The organic extract was dried over anhydrous sodium sulphate and evaporated to yield the product as a yellow oil (yield: $16.6 \mathrm{~g}, 85 \%) .{ }^{1} \mathrm{H} \mathrm{NMR}\left(\mathrm{CDCl}_{3}, 400.13 \mathrm{MHz}\right) 5.72-$ 5.57 (m, 3H, cyclohexadiene-CH=), $3.17\left(\mathrm{~s}, 2 \mathrm{H}, \mathrm{CH}_{2}-\mathrm{N}\right), 2.70-2.63(\mathrm{~m}, 4 \mathrm{H}$, cyclohexadiene- $\mathrm{CH}_{2}$ ), 1.19 (b, $2 \mathrm{H}, \mathrm{NH}_{2}$ ).

Synthesis of 1-methylamide-(4-formylphenoxyacetyl)-1,4-cyclohexadiene (1)

Bromoacetyl bromide (0.14 g, $0.68 \mathrm{mmol})$ was dissolved in dichloromethane $(5 \mathrm{ml})$ and cooled in an ice bath to $0^{\circ} \mathrm{C}$. A solution of 1-methylamine-1,4-cyclohexadiene $(0.20$ g, $1.83 \mathrm{mmol})$ in dichloromethane $(5 \mathrm{ml})$ was added dropwise and the reaction mixture was stirred at $0^{\circ} \mathrm{C}$ for $1 \mathrm{~h}$. Distilled water $(20 \mathrm{ml})$ was added to quench the reaction and the product was extracted with ethyl acetate $(3 \times 20 \mathrm{ml})$. The organic layer was washed successively with $1 \mathrm{M} \mathrm{HCl}$ solution (50 ml) and water (50 ml) and dried over anhydrous sodium sulphate. The solvent was removed to yield 1-methylamide-(bromomethyl)-1,4cyclohexadiene as a white solid. 
1-Methylamide-(bromomethyl)-1,4-cyclohexadiene (0.34 g, $1.47 \mathrm{mmol}$ ) was added to a stirring mixture of 4-hydroxybenzaldehyde (0.18 g, $1.47 \mathrm{mmol})$ and potassium carbonate $(0.31 \mathrm{~g}, 2.24 \mathrm{mmol})$ in acetone $(20 \mathrm{ml})$. The reaction is stirred at room temperature for $16 \mathrm{~h}$. Water $(50 \mathrm{ml})$ was added and the reaction mixture was extracted with dichloromethane ( $3 \times 20 \mathrm{ml})$. The organic fractions were combined, dried over sodium sulphate and concentrated under reduced pressure to yield a yellow oil, which crystallised in vacuo. The product was further purified using flash column chromatography using chloroform:acetonitrile 8:2 (yield: 0.37 g, 93\%). ${ }^{1} \mathrm{H}$ NMR $\left(\mathrm{CDCl}_{3}\right.$, $400.13 \mathrm{MHz}) 9.92$ (s, 1H, -CHO), 7.91 (d, 2H, benzaldehyde-ArH, ${ }^{3} J_{\mathrm{HH}}=9.0 \mathrm{~Hz}$ ), 7.15 (d, $2 \mathrm{H}$, benzaldehyde-ArH, ${ }^{3} J_{\mathrm{HH}}=9.0 \mathrm{~Hz}$ ), $6.50\left(\mathrm{~b}, 1 \mathrm{H},-\mathrm{CON}-\mathrm{H},{ }^{3} J_{\mathrm{HH}}=3.6 \mathrm{~Hz}\right.$ ), 5.70$5.62(\mathrm{~m}, 3 \mathrm{H}$, cyclohexadiene- $\mathrm{CH}=)$, $3.92\left(\mathrm{~s}, 2 \mathrm{H},-\mathrm{CH}_{2} \mathrm{O}-\right), 3.86\left(\mathrm{~s}, 2 \mathrm{H},-\mathrm{CH}_{2}-\mathrm{NH},{ }^{3} \mathrm{~J}_{\mathrm{HH}}=\right.$ 3.6 Hz), 2.70-2.63 (m, 4H, cyclohexadiene- $\mathrm{CH}_{2}$ ).

Synthesis of (4-formylphenoxyacetyl- $\eta^{6}$-benzyl amide)ruthenium dichloride dimer (2)

Ruthenium(III) chloride hydrate (200 mg, $0.77 \mathrm{mmol}$ ) was purified by repeatedly dissolving in methanol $(20 \mathrm{ml})$ and evaporating in vacuo. The purified ruthenium(III) chloride residue was dissolved in ethanol (20 ml) and $\mathbf{1}(720 \mathrm{mg}, 3.32 \mathrm{mmol})$ was added. The solution was degassed and refluxed at $85^{\circ} \mathrm{C}$ for $16 \mathrm{~h}$ under nitrogen. On completion, the reaction mixture was cooled and the brown precipitate was filtered, washed with cold chloroform (5 ml) and diethyl ether (25 ml) and dried in vacuo (yield: $246 \mathrm{mg}, 73 \%) .{ }^{1} \mathrm{H}$ NMR (DMSO-d $\left.d^{6}, 400.13 \mathrm{MHz}\right) 9.89$ (s, 1H, -CHO), 8.67 (t, $1 \mathrm{H},-\mathrm{CON}-H,{ }^{3} J_{\mathrm{HH}}=1.4$ $\mathrm{Hz}$ ), 7.88 (d, 2H, benzaldehyde-ArH, ${ }^{3} J_{\mathrm{HH}}=6.0 \mathrm{~Hz}$ ), 7.17 (d, 2H, benzaldehyde-ArH, ${ }^{3} J_{\mathrm{HH}}=6.0 \mathrm{~Hz}$ ), $6.75\left(\mathrm{t}, 2 \mathrm{H}, \eta^{6}\right.$-phenyl-ArH, $\left.{ }^{3} J_{\mathrm{HH}}=3.6 \mathrm{~Hz}\right), 5.89$ (d, 2H, $\eta^{6}$-phenyl-ArH, 
${ }^{3} J_{\mathrm{HH}}=3.6 \mathrm{~Hz}$ ), $5.81\left(\mathrm{t}, 1 \mathrm{H}, \eta^{6}-\right.$ phenyl-ArH, $\left.{ }^{3} J_{\mathrm{HH}}=3.6 \mathrm{~Hz}\right), 4.72\left(\mathrm{~s}, 2 \mathrm{H},-\mathrm{CH}_{2} \mathrm{O}-\right), 3.25$ (s, $\left.2 \mathrm{H},-\mathrm{CH}_{2}-\mathrm{NH},{ }^{3} \mathrm{~J}_{\mathrm{HH}}=1.4 \mathrm{~Hz}\right)$.

\section{Synthesis of RAPTA-FORM (3)}

Ruthenium dimer 2 (134 mg, $0.15 \mathrm{mmol}$ ) was dissolved in degassed DMF (5 ml). The pta ligand (50 mg, $0.32 \mathrm{mmol}$ ) was added and the solution was stirred for a further 30 min at room temperature. On completion, the solution turned red-orange. The reaction mixture was concentrated to $10 \%$ of its original volume in vacuo and cold diethyl ether was added to precipitate the product. The product was recrystallised from DMF/diethyl ether to yield an orange precipitate (yield: $144 \mathrm{mg}, 90 \%) .{ }^{1} \mathrm{H}$ NMR (DMSO- $d^{6}, 400.13$ MHz) 9.77 (s, 1H, -CHO), 8.55 (t, $\left.1 \mathrm{H},-\mathrm{CON}-H,{ }^{3} J_{\mathrm{HH}}=1.4 \mathrm{~Hz}\right), 7.80$ (d, $2 \mathrm{H}$, benzaldehyde-ArH, $\left.{ }^{3} \mathrm{~J}_{\mathrm{HH}}=6.0 \mathrm{~Hz}\right), 7.05\left(\mathrm{~d}, 2 \mathrm{H}\right.$, benzaldehyde-ArH, $\left.{ }^{3} \mathrm{~J}_{\mathrm{HH}}=6.0 \mathrm{~Hz}\right), 6.64$ (t, 2H, $\eta^{6}$-phenyl-ArH, ${ }^{3} J_{\mathrm{HH}}=3.6 \mathrm{~Hz}$ ), 5.89 (d, 2H, $\eta^{6}$-phenyl-ArH, ${ }^{3} J_{\mathrm{HH}}=3.6 \mathrm{~Hz}$ ), 5.70 (t, $1 \mathrm{H}, \eta^{6}$-phenyl-ArH, $\left.{ }^{3} J_{\mathrm{HH}}=3.6 \mathrm{~Hz}\right), 4.62\left(\mathrm{~s}, 2 \mathrm{H},-\mathrm{CH}_{2} \mathrm{O}-\right), 4.42\left(\mathrm{~s}, 6 \mathrm{H}, \mathrm{pta}-\mathrm{CH}_{2}\right), 4.19$ (s, 6H, pta- $\mathrm{CH}_{2}$ ), 4.10 (s, 2H, $-\mathrm{CH}_{2}-\mathrm{NH},{ }^{3} J_{\mathrm{HH}}=1.4 \mathrm{~Hz}$ ). ${ }^{31} \mathrm{P}\left\{{ }^{1} \mathrm{H}\right\}-\mathrm{NMR}$ (DMSO-d $d^{6}$, 161.97 MHz) -31.19 (s). ${ }^{31} \mathrm{P}\left\{{ }^{1} \mathrm{H}\right\}-\mathrm{NMR}\left(\mathrm{D}_{2} \mathrm{O}, 161.97 \mathrm{MHz}\right)-31.98$ (s). nESI-MS $\left(\mathrm{H}_{2} \mathrm{O}\right.$, +ve mode) m/z: $598.7[\mathrm{M}+\mathrm{H}]^{+}, 563.4[\mathrm{M}-\mathrm{Cl}]^{+}$. Anal. $\left(\mathrm{C}_{22} \mathrm{H}_{27} \mathrm{Cl}_{2} \mathrm{~N}_{4} \mathrm{O}_{3} \mathrm{PRu} \cdot \mathrm{H}_{2} \mathrm{O}\right) \mathrm{C}$ 42.87, H 4.74, N 9.09, Found C 42.65, H 4.55, N 9.51.

\section{Conjugation of rHSA with RAPTA}

The supplied rHSA solution was purified by dialysis $(3 \mathrm{ml}, 50 \mathrm{mg} / \mathrm{ml})$ using the Slide-A-Lyzer (Pierce) against the modification buffer (PBS, $\mathrm{pH} 7.4$ ) in accordance to the manufacturer's instructions. The concentration of the purified protein was determined 
using the Bradford assay (Bio-Rad) using BSA as the reference protein. The purified protein $(2.5 \mathrm{ml}, 41.5 \mathrm{mg}$ protein $/ \mathrm{ml})$ was shaken with a solution of SHTH $(4.7 \mathrm{mg}, 10$ eq.) in DMF (50 $\mu \mathrm{l})$ for $16 \mathrm{~h}$ at room temperature such that the DMF volume did not exceed 5\% v/v. On completion, the protein reaction mixture was dialysed using the SlideA-Lyzer against the conjugation buffer (100 mM MES, 0.9\% NaCl, pH 6.0). The concentration of the modified protein was determined using the Bradford assay. The modified protein solution ( $1 \mathrm{ml}, 34.3 \mathrm{mg}$ protein/ml) was added to solutions of $3(1 \mathrm{mM}$, $7.5 \mathrm{ml}, 10$ eq.) and shaken for $6 \mathrm{~h}$ at room temperature. On completion, the protein mixture solution was concentrated using MWCO/10K centrifugal units (Millipore), desalted by gel-filtration spin columns (Zeba) and reconcentrated using MWCO/10K centrifugal units against PBS. The concentration of conjugated rHSA-RAPTA in PBS was determined using Bradford assay to be $48.6 \mathrm{mg}$ protein $/ \mathrm{ml}$. 


\section{UV-vis characterization}

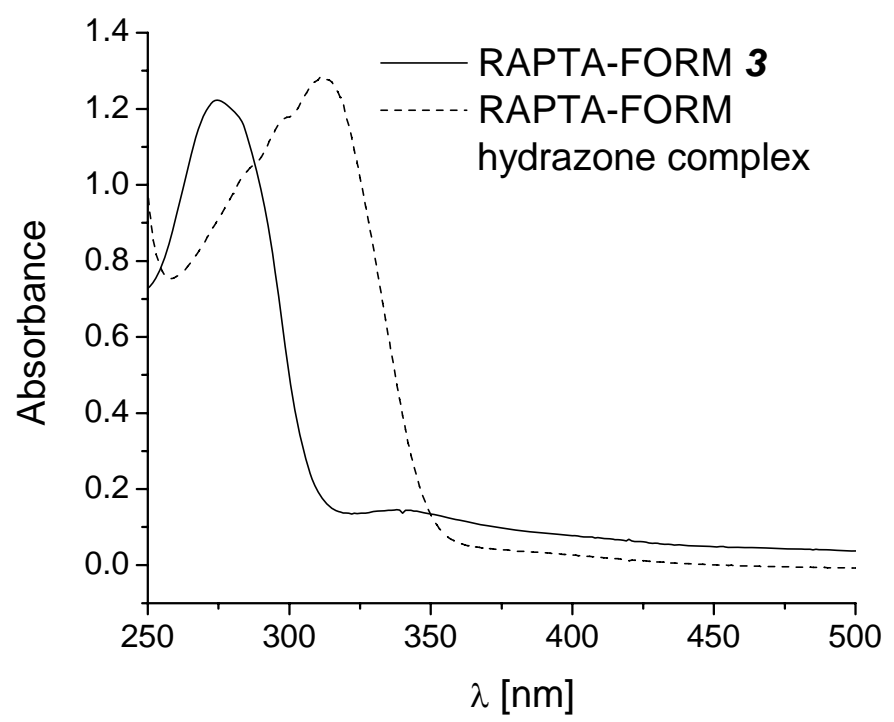

Figure S1. UV-vis spectra of RAPTA-FORM 3 (solid line) and its hydrazone complex with benzoyl hydrazone (dashed line).

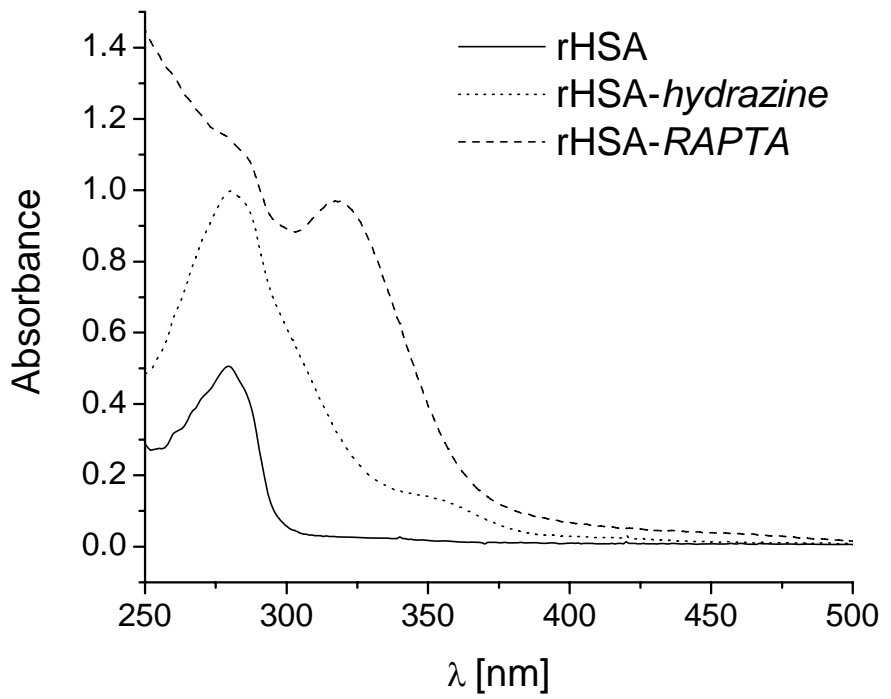

Figure S2. UV-vis spectra of rHSA (solid line), rHSA modified with hydrazine (dotted line) and rHSA conjugated to RAPTA (dashed line); absorbance maxima at $317 \mathrm{~nm}$ on rHSA-RAPTA corresponds to presence of hydrazone bonds. 


\section{MALDI-TOF MS analyses}

The purified rHSA samples were characterized by MALDI-ToF-MS using an Applied Biosystems 4800 MALDI ToF/ToF mass spectrometer. The samples were prepared using the dried droplet method with freshly prepared sinapinic acid $(10 \mathrm{mg} / \mathrm{ml}$ in $50 \%$ MeCN/0.1\% TFA) as matrix solutions. The protein samples and matrix were mixed in $1: 10 \mathrm{v} / \mathrm{v}$, plated on the sample target and allowed to evaporate in air. The mass spectra were recorded in the range from to 30000 to $100000 \mathrm{~m} / \mathrm{z}$ in the positive ion mode. The instrument was calibrated with Insulin, Ubiquitin, Cytochrome-C and Alpha-synuclein A30P. The measured rHSA peak is 66,331 Da (expected: 66,357 Da) corresponding to an uncertainty of $0.04 \%$.

\section{Structural characterization of (1) and (3) in the solid state}

Graphical representations are given in Figures 2 and S2 with bond distances and angles given in Tables S1 and S2. Data collection was performed on the Bruker-Nonius KappaCDD diffractometer. The unit cell and orientation matrix was determined by indexing reflections measured from a sampling of scan and analyzed with DirAX. ${ }^{5}$ Data collection was performed by scanning reflections from the entire Ewald sphere using the program CollectCCD, and data reduction using EvalCCD. ${ }^{6,7}$ Structure solution was performed using SHELXS-97, and the structure refined by full-matrix least-squares refinement (against $F^{2}$ ) using SHELXTL software. ${ }^{8}$ All non-hydrogen atoms were refined anisotropically while hydrogen atoms were placed in their geometrically generated positions and refined using the riding model. Twinning was modelled using the TWIN

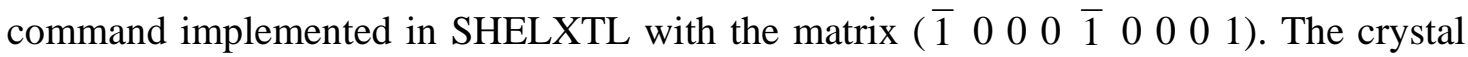
lattice was solvated by disordered DMF molecules which occupied the unit cell at $50 \%$ 
occupancy level. Restraints were applied on all carbon and oxygen atoms, as well as disordered DMF solvent molecules, using DELU and SIMU implemented in SHELXTL. A multi-scan absorption correction based on a semi-empirical method was applied using the SADABS. ${ }^{9}$ Graphical representations of the structures were made with Diamond. ${ }^{10}$

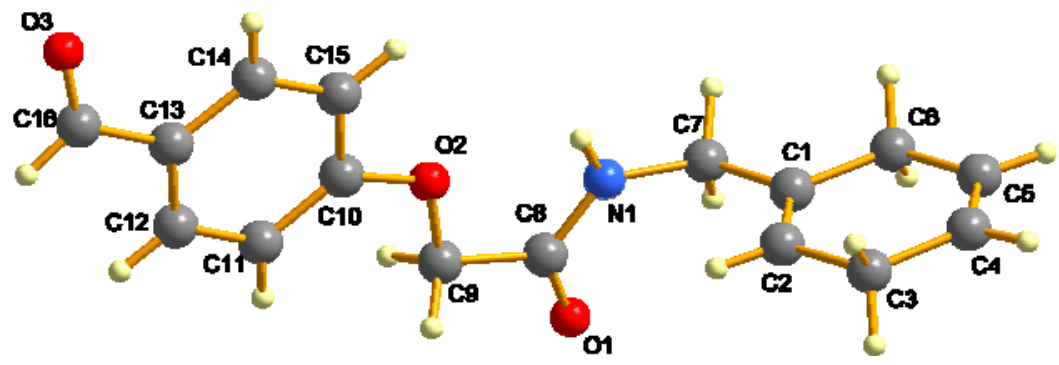

Figure S2. Ball and stick representation of $\mathbf{1}$; atoms are spheres of arbitrary diameter. 
Table S1. Bond lengths $[\AA]$ and angles $\left[^{\circ}\right]$ for $\mathbf{1}$.

\begin{tabular}{|c|c|}
\hline $\mathrm{N}(1)-\mathrm{C}(8)$ & $1.344(2)$ \\
\hline $\mathrm{N}(1)-\mathrm{C}(7)$ & $1.457(2)$ \\
\hline $\mathrm{O}(1)-\mathrm{C}(8)$ & $1.2350(19)$ \\
\hline $\mathrm{O}(2)-\mathrm{C}(10)$ & $1.364(2)$ \\
\hline $\mathrm{O}(2)-\mathrm{C}(9)$ & $1.4420(19)$ \\
\hline $\mathrm{O}(3)-\mathrm{C}(16)$ & $1.226(2)$ \\
\hline $\mathrm{C}(1)-\mathrm{C}(2)$ & $1.334(2)$ \\
\hline$C(1)-C(6)$ & $1.509(2)$ \\
\hline $\mathrm{C}(1)-\mathrm{C}(7)$ & $1.517(2)$ \\
\hline $\mathrm{C}(2)-\mathrm{C}(3)$ & $1.508(2)$ \\
\hline C(3)-C(4) & 1.503(3) \\
\hline$C(4)-C(5)$ & $1.329(3)$ \\
\hline$C(5)-C(6)$ & $1.500(2)$ \\
\hline C(8)-C(9) & $1.521(2)$ \\
\hline $\mathrm{C}(10)-\mathrm{C}(11)$ & $1.395(2)$ \\
\hline C(10)-C(15) & $1.410(2)$ \\
\hline C(11)-C(12) & $1.397(2)$ \\
\hline C(12)-C(13) & $1.406(2)$ \\
\hline C(13)-C(14) & $1.403(2)$ \\
\hline C(13)-C(16) & $1.466(2)$ \\
\hline C(14)-C(15) & $1.378(2)$ \\
\hline $\mathrm{C}(8)-\mathrm{N}(1)-\mathrm{C}(7)$ & $122.60(14)$ \\
\hline $\mathrm{C}(10)-\mathrm{O}(2)-\mathrm{C}(9)$ & 118.67(13) \\
\hline $\mathrm{C}(2)-\mathrm{C}(1)-\mathrm{C}(6)$ & 122.63(16) \\
\hline $\mathrm{C}(2)-\mathrm{C}(1)-\mathrm{C}(7)$ & $124.58(16)$ \\
\hline $\mathrm{C}(6)-\mathrm{C}(1)-\mathrm{C}(7)$ & 112.79(14) \\
\hline$C(1)-C(2)-C(3)$ & 123.69(17) \\
\hline $\mathrm{C}(4)-\mathrm{C}(3)-\mathrm{C}(2)$ & $113.00(15)$ \\
\hline C(5)-C(4)-C(3) & 123.68(17) \\
\hline$C(4)-C(5)-C(6)$ & $123.23(18)$ \\
\hline $\mathrm{C}(5)-\mathrm{C}(6)-\mathrm{C}(1)$ & 113.76(15) \\
\hline $\mathrm{N}(1)-\mathrm{C}(7)-\mathrm{C}(1)$ & $115.10(14)$ \\
\hline $\mathrm{O}(1)-\mathrm{C}(8)-\mathrm{N}(1)$ & 124.59(16) \\
\hline
\end{tabular}




$\begin{array}{ll}\text { O(1)-C(8)-C(9) } & 119.02(15) \\ \text { N(1)-C(8)-C(9) } & 116.38(14) \\ \text { O(2)-C(9)-C(8) } & 108.57(13) \\ \text { O(2)-C(10)-C(11) } & 124.88(15) \\ \text { O(2)-C(10)-C(15) } & 113.93(15) \\ \mathrm{C}(11)-C(10)-C(15) & 121.19(16) \\ \mathrm{C}(10)-\mathrm{C}(11)-\mathrm{C}(12) & 118.02(15) \\ \mathrm{C}(11)-\mathrm{C}(12)-\mathrm{C}(13) & 121.36(16) \\ \mathrm{C}(14)-\mathrm{C}(13)-\mathrm{C}(12) & 119.41(16) \\ \mathrm{C}(14)-\mathrm{C}(13)-\mathrm{C}(16) & 120.77(15) \\ \mathrm{C}(12)-\mathrm{C}(13)-\mathrm{C}(16) & 119.77(16) \\ \mathrm{C}(15)-\mathrm{C}(14)-\mathrm{C}(13) & 119.97(15) \\ \mathrm{C}(14)-\mathrm{C}(15)-\mathrm{C}(10) & 120.04(16) \\ \mathrm{O}(3)-\mathrm{C}(16)-\mathrm{C}(13) & 124.93(17)\end{array}$


Table S2. Bond lengths $[\AA]$ and angles $\left[^{\circ}\right]$ for 3.

Table 3. Bond lengths $[\AA]$ and angles $\left[{ }^{\circ}\right]$ for pd427.

\begin{tabular}{|c|c|}
\hline $\mathrm{Ru}(1)-\mathrm{C}(5)$ & $2.175(10)$ \\
\hline $\mathrm{Ru}(1)-\mathrm{C}(4)$ & $2.178(10)$ \\
\hline $\mathrm{Ru}(1)-\mathrm{C}(6)$ & $2.224(9)$ \\
\hline $\mathrm{Ru}(1)-\mathrm{C}(3)$ & $2.229(9)$ \\
\hline $\mathrm{Ru}(1)-\mathrm{C}(1)$ & $2.275(9)$ \\
\hline $\mathrm{Ru}(1)-\mathrm{C}(2)$ & $2.277(9)$ \\
\hline $\mathrm{Ru}(1)-\mathrm{P}(1)$ & $2.309(4)$ \\
\hline $\mathrm{Ru}(1)-\mathrm{Cl}(2)$ & $2.432(4)$ \\
\hline $\mathrm{Ru}(1)-\mathrm{Cl}(1)$ & $2.442(4)$ \\
\hline$P(1)-C(18)$ & $1.821(16)$ \\
\hline $\mathrm{P}(1)-\mathrm{C}(19)$ & $1.834(14)$ \\
\hline $\mathrm{P}(1)-\mathrm{C}(17)$ & $1.878(17)$ \\
\hline $\mathrm{O}(1)-\mathrm{C}(8)$ & $1.224(18)$ \\
\hline $\mathrm{O}(2)-\mathrm{C}(10)$ & $1.394(18)$ \\
\hline $\mathrm{O}(2)-\mathrm{C}(9)$ & $1.394(16)$ \\
\hline $\mathrm{O}(3 \mathrm{~A})-\mathrm{C}(16)$ & $1.21(3)$ \\
\hline $\mathrm{O}(3 \mathrm{~B})-\mathrm{C}(16)$ & $1.20(5)$ \\
\hline $\mathrm{N}(1)-\mathrm{C}(22)$ & $1.429(19)$ \\
\hline $\mathrm{N}(1)-\mathrm{C}(17)$ & $1.44(2)$ \\
\hline$N(1)-C(21)$ & $1.47(2)$ \\
\hline$N(2)-C(18)$ & $1.46(2)$ \\
\hline$N(2)-C(20)$ & $1.47(2)$ \\
\hline $\mathrm{N}(2)-\mathrm{C}(22)$ & $1.48(2)$ \\
\hline $\mathrm{N}(3)-\mathrm{C}(19)$ & $1.45(2)$ \\
\hline $\mathrm{N}(3)-\mathrm{C}(20)$ & $1.48(2)$ \\
\hline$N(3)-C(21)$ & $1.48(2)$ \\
\hline $\mathrm{N}(4)-\mathrm{C}(8)$ & $1.376(19)$ \\
\hline $\mathrm{N}(4)-\mathrm{C}(7)$ & $1.48(2)$ \\
\hline $\mathrm{C}(1)-\mathrm{C}(2)$ & 1.3900 \\
\hline$C(1)-C(6)$ & 1.3900 \\
\hline $\mathrm{C}(1)-\mathrm{C}(7)$ & $1.525(16)$ \\
\hline$C(2)-C(3)$ & 1.3900 \\
\hline
\end{tabular}




\begin{tabular}{|c|c|}
\hline $\mathrm{C}(3)-\mathrm{C}(4)$ & 1.3900 \\
\hline$C(4)-C(5)$ & 1.3900 \\
\hline$C(5)-C(6)$ & 1.3900 \\
\hline $\mathrm{C}(8)-\mathrm{C}(9)$ & $1.57(2)$ \\
\hline $\mathrm{C}(10)-\mathrm{C}(11)$ & $1.38(2)$ \\
\hline C(10)-C(15) & $1.40(2)$ \\
\hline $\mathrm{C}(11)-\mathrm{C}(12)$ & $1.41(2)$ \\
\hline C(12)-C(13) & $1.38(2)$ \\
\hline C(13)-C(14) & $1.38(2)$ \\
\hline$C(13)-C(16)$ & $1.51(2)$ \\
\hline C(14)-C(15) & $1.43(2)$ \\
\hline N(30)-C(30) & $1.341(18)$ \\
\hline N(30)-C(31) & $1.46(4)$ \\
\hline N(30)-C(32) & $1.50(4)$ \\
\hline $\mathrm{O}(30)-\mathrm{C}(30)$ & $1.248(19)$ \\
\hline $\mathrm{C}(5)-\mathrm{Ru}(1)-\mathrm{C}(4)$ & $37.24(16)$ \\
\hline C(5)-Ru(1)-C(6) & 36.81(15) \\
\hline $\mathrm{C}(4)-\mathrm{Ru}(1)-\mathrm{C}(6)$ & $66.3(2)$ \\
\hline C(5)-Ru(1)-C(3) & $66.3(2)$ \\
\hline $\mathrm{C}(4)-\mathrm{Ru}(1)-\mathrm{C}(3)$ & $36.75(15)$ \\
\hline $\mathrm{C}(6)-\mathrm{Ru}(1)-\mathrm{C}(3)$ & 77.3(2) \\
\hline $\mathrm{C}(5)-\mathrm{Ru}(1)-\mathrm{C}(1)$ & $65.5(2)$ \\
\hline$C(4)-R u(1)-C(1)$ & $77.2(2)$ \\
\hline $\mathrm{C}(6)-\mathrm{Ru}(1)-\mathrm{C}(1)$ & $35.97(13)$ \\
\hline $\mathrm{C}(3)-\mathrm{Ru}(1)-\mathrm{C}(1)$ & 64.62(19) \\
\hline $\mathrm{C}(5)-\mathrm{Ru}(1)-\mathrm{C}(2)$ & $77.2(2)$ \\
\hline C(4)-Ru(1)-C(2) & $65.4(2)$ \\
\hline $\mathrm{C}(6)-\mathrm{Ru}(1)-\mathrm{C}(2)$ & 64.66(19) \\
\hline $\mathrm{C}(3)-\mathrm{Ru}(1)-\mathrm{C}(2)$ & 35.91(13) \\
\hline $\mathrm{C}(1)-\mathrm{Ru}(1)-\mathrm{C}(2)$ & $35.56(13)$ \\
\hline $\mathrm{C}(5)-\mathrm{Ru}(1)-\mathrm{P}(1)$ & $91.0(2)$ \\
\hline $\mathrm{C}(4)-\mathrm{Ru}(1)-\mathrm{P}(1)$ & $94.6(3)$ \\
\hline $\mathrm{C}(6)-\mathrm{Ru}(1)-\mathrm{P}(1)$ & $115.1(2)$ \\
\hline C(3)-Ru(1)-P(1) & 123.0(3) \\
\hline C(1)-Ru(1)-P(1) & $150.8(2)$ \\
\hline
\end{tabular}




\begin{tabular}{|c|c|}
\hline $\mathrm{C}(2)-\mathrm{Ru}(1)-\mathrm{P}(1)$ & $158.8(3)$ \\
\hline $\mathrm{C}(5)-\mathrm{Ru}(1)-\mathrm{Cl}(2)$ & 118.3(3) \\
\hline $\mathrm{C}(4)-\mathrm{Ru}(1)-\mathrm{Cl}(2)$ & 155.5(3) \\
\hline $\mathrm{C}(6)-\mathrm{Ru}(1)-\mathrm{Cl}(2)$ & $93.0(2)$ \\
\hline $\mathrm{C}(3)-\mathrm{Ru}(1)-\mathrm{Cl}(2)$ & $154.7(3)$ \\
\hline $\mathrm{C}(1)-\mathrm{Ru}(1)-\mathrm{Cl}(2)$ & $93.8(2)$ \\
\hline $\mathrm{C}(2)-\mathrm{Ru}(1)-\mathrm{Cl}(2)$ & 118.9(3) \\
\hline $\mathrm{P}(1)-\mathrm{Ru}(1)-\mathrm{Cl}(2)$ & $82.21(15)$ \\
\hline $\mathrm{C}(5)-\mathrm{Ru}(1)-\mathrm{Cl}(1)$ & 154.6(3) \\
\hline $\mathrm{C}(4)-\mathrm{Ru}(1)-\mathrm{Cl}(1)$ & 117.6(3) \\
\hline $\mathrm{C}(6)-\mathrm{Ru}(1)-\mathrm{Cl}(1)$ & $157.4(2)$ \\
\hline $\mathrm{C}(3)-\mathrm{Ru}(1)-\mathrm{Cl}(1)$ & $93.7(2)$ \\
\hline $\mathrm{C}(1)-\mathrm{Ru}(1)-\mathrm{Cl}(1)$ & $121.4(2)$ \\
\hline $\mathrm{C}(2)-\mathrm{Ru}(1)-\mathrm{Cl}(1)$ & $95.8(2)$ \\
\hline $\mathrm{P}(1)-\mathrm{Ru}(1)-\mathrm{Cl}(1)$ & $87.28(14)$ \\
\hline $\mathrm{Cl}(2)-\mathrm{Ru}(1)-\mathrm{Cl}(1)$ & 86.61(14) \\
\hline C(18)-P(1)-C(19) & $97.5(8)$ \\
\hline $\mathrm{C}(18)-\mathrm{P}(1)-\mathrm{C}(17)$ & 98.2(8) \\
\hline C(19)-P(1)-C(17) & 97.1(8) \\
\hline $\mathrm{C}(18)-\mathrm{P}(1)-\mathrm{Ru}(1)$ & $120.0(5)$ \\
\hline $\mathrm{C}(19)-\mathrm{P}(1)-\mathrm{Ru}(1)$ & $116.4(5)$ \\
\hline $\mathrm{C}(17)-\mathrm{P}(1)-\mathrm{Ru}(1)$ & $122.5(6)$ \\
\hline $\mathrm{C}(10)-\mathrm{O}(2)-\mathrm{C}(9)$ & $118.2(11)$ \\
\hline $\mathrm{C}(22)-\mathrm{N}(1)-\mathrm{C}(17)$ & 111.3(14) \\
\hline $\mathrm{C}(22)-\mathrm{N}(1)-\mathrm{C}(21)$ & 108.1(13) \\
\hline $\mathrm{C}(17)-\mathrm{N}(1)-\mathrm{C}(21)$ & 112.1(13) \\
\hline C(18)-N(2)-C(20) & 111.1(13) \\
\hline $\mathrm{C}(18)-\mathrm{N}(2)-\mathrm{C}(22)$ & $110.5(13)$ \\
\hline $\mathrm{C}(20)-\mathrm{N}(2)-\mathrm{C}(22)$ & $108.4(13)$ \\
\hline C(19)-N(3)-C(20) & $111.9(15)$ \\
\hline C(19)-N(3)-C(21) & $110.0(14)$ \\
\hline $\mathrm{C}(20)-\mathrm{N}(3)-\mathrm{C}(21)$ & $108.4(13)$ \\
\hline $\mathrm{C}(8)-\mathrm{N}(4)-\mathrm{C}(7)$ & $121.4(13)$ \\
\hline $\mathrm{C}(2)-\mathrm{C}(1)-\mathrm{C}(6)$ & 120.0 \\
\hline C(2)-C(1)-C(7) & 119.8(8) \\
\hline$C(6)-C(1)-C(7)$ & $120.1(8)$ \\
\hline
\end{tabular}




\begin{tabular}{|c|c|}
\hline $\mathrm{C}(2)-\mathrm{C}(1)-\mathrm{Ru}(1)$ & 72.3(3) \\
\hline $\mathrm{C}(6)-\mathrm{C}(1)-\mathrm{Ru}(1)$ & $70.0(3)$ \\
\hline $\mathrm{C}(7)-\mathrm{C}(1)-\mathrm{Ru}(1)$ & $126.6(8)$ \\
\hline$C(1)-C(2)-C(3)$ & 120.0 \\
\hline $\mathrm{C}(1)-\mathrm{C}(2)-\mathrm{Ru}(1)$ & 72.1(3) \\
\hline$C(3)-C(2)-R u(1)$ & $70.2(3)$ \\
\hline$C(4)-C(3)-C(2)$ & 120.0 \\
\hline $\mathrm{C}(4)-\mathrm{C}(3)-\mathrm{Ru}(1)$ & 69.6(3) \\
\hline $\mathrm{C}(2)-\mathrm{C}(3)-\mathrm{Ru}(1)$ & 73.9(3) \\
\hline$C(5)-C(4)-C(3)$ & 120.0 \\
\hline C(5)-C(4)-Ru(1) & 71.3(3) \\
\hline $\mathrm{C}(3)-\mathrm{C}(4)-\mathrm{Ru}(1)$ & 73.6(3) \\
\hline$C(4)-C(5)-C(6)$ & 120.0 \\
\hline $\mathrm{C}(4)-\mathrm{C}(5)-\mathrm{Ru}(1)$ & $71.5(3)$ \\
\hline$C(6)-C(5)-R u(1)$ & $73.5(3)$ \\
\hline$C(5)-C(6)-C(1)$ & 120.0 \\
\hline$C(5)-C(6)-R u(1)$ & 69.7(3) \\
\hline $\mathrm{C}(1)-\mathrm{C}(6)-\mathrm{Ru}(1)$ & $74.0(3)$ \\
\hline $\mathrm{N}(4)-\mathrm{C}(7)-\mathrm{C}(1)$ & $112.9(11)$ \\
\hline $\mathrm{O}(1)-\mathrm{C}(8)-\mathrm{N}(4)$ & $125.3(14)$ \\
\hline $\mathrm{O}(1)-\mathrm{C}(8)-\mathrm{C}(9)$ & $121.2(13)$ \\
\hline $\mathrm{N}(4)-\mathrm{C}(8)-\mathrm{C}(9)$ & 113.5(13) \\
\hline $\mathrm{O}(2)-\mathrm{C}(9)-\mathrm{C}(8)$ & 108.7(11) \\
\hline $\mathrm{C}(11)-\mathrm{C}(10)-\mathrm{O}(2)$ & $125.7(13)$ \\
\hline$C(11)-C(10)-C(15)$ & $121.2(15)$ \\
\hline $\mathrm{O}(2)-\mathrm{C}(10)-\mathrm{C}(15)$ & 113.2(13) \\
\hline$C(10)-C(11)-C(12)$ & $119.4(15)$ \\
\hline$C(13)-C(12)-C(11)$ & $120.7(15)$ \\
\hline $\mathrm{C}(12)-\mathrm{C}(13)-\mathrm{C}(14)$ & 120.1(16) \\
\hline $\mathrm{C}(12)-\mathrm{C}(13)-\mathrm{C}(16)$ & 119.7(16) \\
\hline$C(14)-C(13)-C(16)$ & $120.2(16)$ \\
\hline$C(13)-C(14)-C(15)$ & $119.9(15)$ \\
\hline$C(10)-C(15)-C(14)$ & $118.7(15)$ \\
\hline $\mathrm{O}(3 \mathrm{~B})-\mathrm{C}(16)-\mathrm{O}(3 \mathrm{~A})$ & 111(3) \\
\hline $\mathrm{O}(3 \mathrm{~B})-\mathrm{C}(16)-\mathrm{C}(13)$ & 124(3) \\
\hline $\mathrm{O}(3 \mathrm{~A})-\mathrm{C}(16)-\mathrm{C}(13)$ & $125(2)$ \\
\hline
\end{tabular}




$\begin{array}{ll}\mathrm{N}(1)-\mathrm{C}(17)-\mathrm{P}(1) & 111.4(12) \\ \mathrm{N}(2)-\mathrm{C}(18)-\mathrm{P}(1) & 112.9(11) \\ \mathrm{N}(3)-\mathrm{C}(19)-\mathrm{P}(1) & 113.6(10) \\ \mathrm{N}(2)-\mathrm{C}(20)-\mathrm{N}(3) & 112.8(13) \\ \mathrm{N}(1)-\mathrm{C}(21)-\mathrm{N}(3) & 114.0(12) \\ \mathrm{N}(1)-\mathrm{C}(22)-\mathrm{N}(2) & 116.0(13) \\ \mathrm{C}(30)-\mathrm{N}(30)-\mathrm{C}(31) & 127(2) \\ \mathrm{C}(30)-\mathrm{N}(30)-\mathrm{C}(32) & 113(2) \\ \mathrm{C}(31)-\mathrm{N}(30)-\mathrm{C}(32) & 120(2) \\ \mathrm{O}(30)-\mathrm{C}(30)-\mathrm{N}(30) & 121(3)\end{array}$




\section{Cell culture and inhibition of cell growth}

Human A2780 and A2780cisR ovarian carcinoma cell lines were obtained from the European Centre of Cell Cultures (ECACC, Salisbury, UK) and maintained in culture as described by the provider. The cells were routinely grown in RPMI 1640 medium containing $10 \%$ fetal calf serum (FCS) and antibiotics at $37^{\circ} \mathrm{C}$ and $6 \% \mathrm{CO}_{2}$. For the evaluation of growth inhibition tests, the cells were seeded in 96-well plates (Costar, Integra Biosciences, Cambridge, USA) and grown for $24 \mathrm{~h}$ in complete medium. Complex 3 and rHSA solutions were diluted directly in culture medium to the required concentration and added to the cell culture for $72 \mathrm{~h}$ incubation. The MTT test was performed for the last $2 \mathrm{~h}$ without changing the culture medium. Briefly, following drug exposure, MTT (Sigma) was added to the cells at the final concentration of $0.2 \mathrm{mg} / \mathrm{ml}$ and incubated for $2 \mathrm{~h}$, then the culture medium was aspirated and the violet formazan precipitate dissolved in $0.1 \mathrm{~N} \mathrm{HCl}$ in 2-propanol. The optical density was quantified at $540 \mathrm{~nm}$ using a multiwell plate reader (iEMS Reader MF, Labsystems, US), and the percentage of surviving cells was calculated from the ratio of absorbance of treated to untreated cells. The $\mathrm{IC}_{50}$ values for the inhibition of cell growth were determined by fitting the plot of the percentage of surviving cells against the drug concentration using a sigmoidal function (Origin v7.5).

\section{References}

(1) Schwartz, D. A.; Abrams, M. J.; Hauser, M. M.; Gaul, F. E.; Larsen, S. K.; Rauh, D.; Zubieta, J. A. Bioconjugate Chem. 1991, 2, 333-336. 
(2) Maxwell, J. R.; Wasdahl, D. A.; Wolfson, A. C.; Stenberg, V. I. J. Med. Chem. 1984, 27, 1565-1570.

(3) Dyson, P. J.; McIndoe, J. S. Inorg. Chim. Acta 2003, 354, 68-74.

(4) Stapleford, K. S. J. J. Chem. Soc. Sect. C 1971, 14, 2580-2582.

(5) Duisenberg, A. J. M. J. Appl. Crystallogr. 1992, 25, 92-96.

(6) Duisenberg, A. J. M.; Kroon-Batenburg, L. M. J.; Schreurs, A. M. M. J. Appl. Crystallogr. 2003, 36, 220-229.

(7) Hooft, R. W. W. CollectCCD, Delft, Netherlands.

(8) Sheldrick, G. M. SHELXTL97, Structure Solution and Refinement Package Göttingen, Germany, 1997.

(9) Sheldrick, G. M. SADABS, Program for Empirical Absorption Correction of Area Detector Data, Göttingen, Germany, 1996.

(10) Crystal Impact GbR, Diamond 3.1d, Bonn, Germany, 2004. 